ХРОМОСФЕРНАЯ КОНДЕНСАЦИЯ

И СУБ-ТЕРАГЕРЦОВОЕ ИЗЛУЧЕНИЕ СОЛНЕЧНЫХ ВСПЫШЕК

\author{
Моргачев А.С. ${ }^{1}$, Цап Ю.Т. ${ }^{2}$, Смирнова В.В. ${ }^{2}$, Моторина Г.Г. ${ }^{3,4}$
}

${ }^{1}$ Нижегородский государственный университет им. Н.И. Лобачевского, Нижний Новгород, Россия

${ }^{2}$ Крымская астрофизическая обсерватория РАН, Научный, Крым, Россия

${ }^{3}$ Главная (Пулковская) астрономическая обсерватория РАН, Санкт-Петербург, Россия ${ }^{4}$ Astronomical Institute ASCR, 25165 Ondrejov, Czech Republic

\title{
CROMOSPHERIC CONDENSATION AND SUB-TERAHETZ EMISSION FROM SOLAR FLARES
}

\author{
Morgachev A.S. ${ }^{1}$, Tsap Yu.T. ${ }^{2}$, Smirnova V.V. ${ }^{2}$, MotorinaG.G. ${ }^{3,4}$ \\ ${ }^{1}$ Lobachevsky University, Nizhny Novgorod, Russia \\ ${ }^{2}$ Crimean Astrophysical Observatory, Nauchny, Crimea, Russia \\ ${ }^{3}$ Pulkovo Observatory of RAS, St. Petersburg, Russia \\ ${ }^{4}$ Astronomical Institute ASCR, 25165 Ondrejov, Czech Republic
}

On the basis of F-CHROMA solar flare model database (https://www.fchroma.org) the temporal evolution of thermal bremsstrahlung in the sub-terahertz frequency range (at 93 and $140 \mathrm{GHz}$ ) from the flare chromosphere and transition region is calculated. The model is based on RADYN numerical code, which describes the response of chromospheric background plasma to the action of a beam of accelerated electrons in the form of a triangular pulse. It has been shown that the positive slope of the spectrum in the 93-400 GHz frequency range during the entire burst is formed. The time profile of the sub-THz emission is quite complex and has several maxima due to gas-dynamic phenomena. The region of low temperature and high plasma density (so-called chromospheric condensation) appears and moves to the region of high altitudes from the $6^{\text {th }}$ to $11^{\text {th }}$ second and then disappears. It determines a complex temporal profile of sub-THz emission which can be used for flare plasma diagnostics.

\section{DOI: $10.31725 / 0552-5829-2020-221-224$}

\section{Введение}

Вопрос о происхождении оптического излучения солнечных и звездных вспышек все еще остается открытым. До сих пор неясно, каким образом происходит нагрев и передача энергии ускоренных частиц в плотные слои атмосферы. В частности, в солнечную фотосферу могут проникнуть только такие электроны, энергия которых превышает сотни кэВ. Однако, принимая во внимание степенной спектр электронов, а также полагая нижнюю пороговую энергию равной 10-20 кэВ, нетрудно прийти к выводу, что на долю таких частиц приходится пренебрежимо малая часть энергии вспышки.

Решение этой проблемы может быть связано с излучением хромосферной конденсации, образующейся в результате газодинамических яв- 
лений [1-3]. В частности, около 40 лет назад в работе [2] на основе численных расчетов было показано, что поток электронов с плотностью энергии $10^{12}$ эрг $\mathrm{cm}^{-2} \mathrm{c}^{-1}$ приводит к формированию солнечной хромосферной конденсации толщиной несколько десятков км, движущейся вниз со скоростью $\sim 10 \mathrm{kм} / \mathrm{c}$. При этом образование плотной $\left(\sim 10^{15} \mathrm{~cm}^{-3}\right)$ и холодной $\left(\sim 10^{4} \mathrm{~K}\right)$ конденсации сопровождается испарением хромосферного вещества с температурой, превышающей миллионы градусов. В настоящее время эти два явления рассматриваются как два фундаментальных процесса вспышечного энерговыделения.

Вплоть до последнего времени исследование свойств хромосферных конденсаций ограничивалось ближним ультрафиолетовым и оптическим диапазоном. В хромосферных линиях, и, прежде всего, в линии $\mathrm{H \alpha}$, они проявляются в виде асимметрии красного крыла линий. Между тем, поскольку вклад в излучение может вносить не только хромосферная конденсация, но окружающая ее плазма, то это существенно затрудняет проведение диагностики этого газодинамического образования. На наш взгляд, именно наблюдения вспышечного суб-ТГц излучения солнечных и звездных вспышек с положительным наклоном спектра, за которое ответственна тепловая плазма [4], позволяют получить важную информацию о свойствах хромосферной конденсации. Изучению этого вопроса и посвящена настоящая работа.

\section{Моделирование суб-ТГц радиоизлучения}

Нами был выполнен расчет теплового тормозного излучения в субтерагерцовом диапазоне для одномерных моделей вспышечной хромосферы и переходной области, полученных на основе численного решения системы нелинейных уравнений газодинамики и переноса излучения в неЛТР приближении с помощью программного кода RADYN [5]. В качестве рабочей была выбрана модель №27 вспышечной хромосферы и переходной области, полученная в рамках международного проекта F-CHROMA (https://www.fchroma.org). Модель описывает отклик невозмущенной солнечной атмосферы типа VAL-C [6] на поток высокоэнергичных нетепловых электронов со степенным энергетическим спектром $\delta$ в виде импульса треугольной формы длительностью 20 сек (рис. 1, правая нижняя панель). В данной модели спектральный индекс $\delta=5$, нижняя граница энергии ускоренных электронов $E_{c}=15$ кэВ и их полный поток $F_{e}=3 \times 10^{11}\left[э р \Gamma^{\times} \times \mathrm{c}^{-}\right.$ $\left.{ }^{1} \times \mathrm{CM}^{-2}\right]$.

Используя модельные высотные зависимости температуры и концентрации плазмы в каждый момент времени, были рассчитаны высотное распределение потока теплового тормозного излучения на частотах 93 и 140 ГГц, временной профиль полного потока излучения модели, а также функция вклада источника (рис.1), используя методику, описанную в работе [7]. 
a)

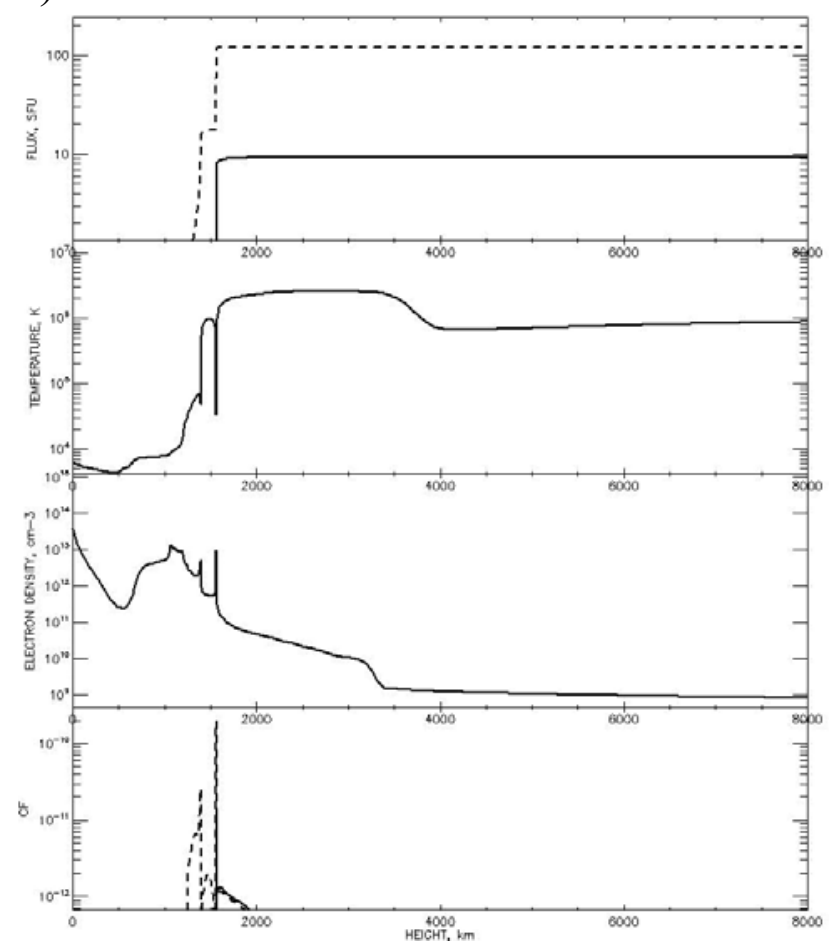

Model $027 \mathrm{E} \_$cut $=15 \mathrm{E}$ _tot $=3.0 \mathrm{e} 11$ index $=5$

time $=8.5$

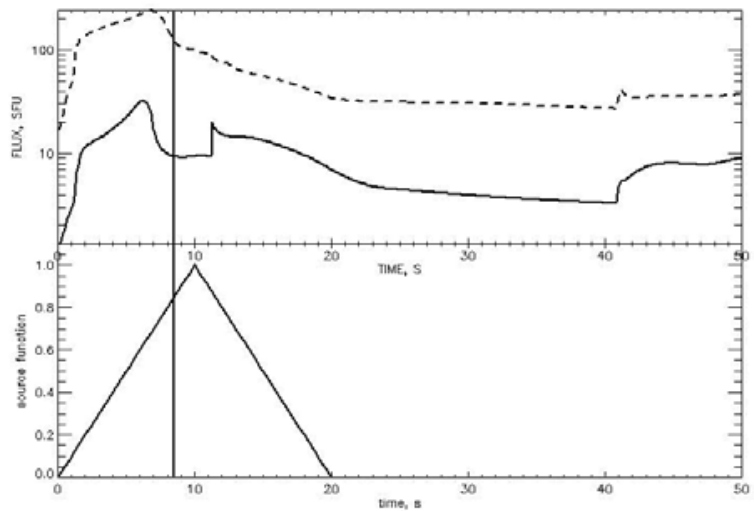

б)

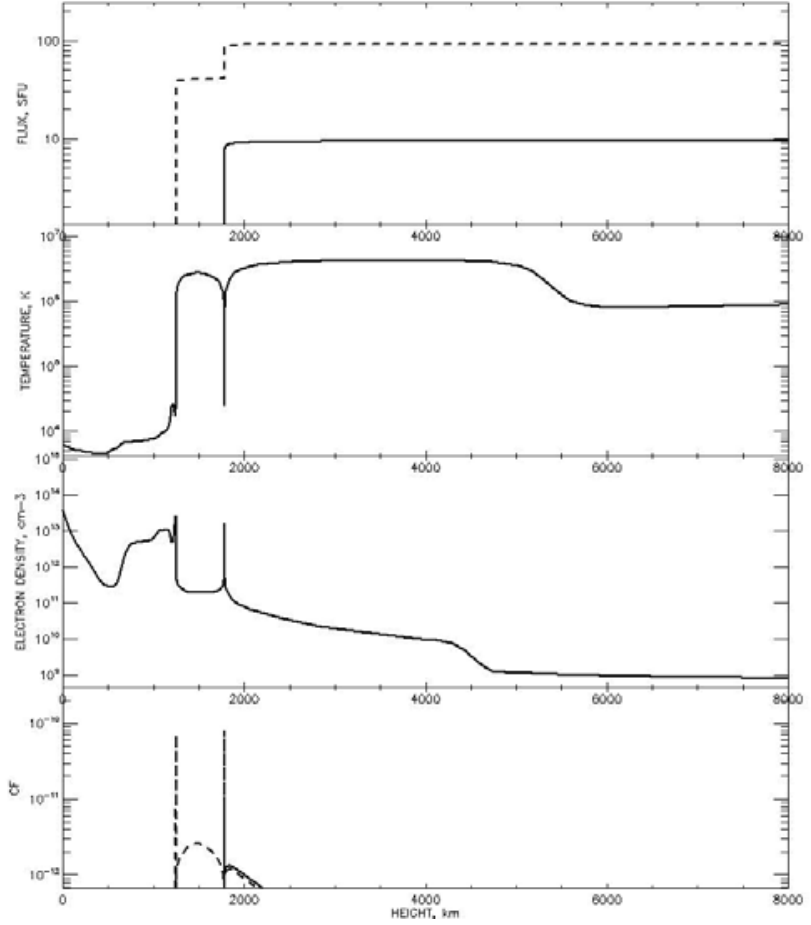

Model 027 E_cut $=15$ E_tot $=3.0$ e 11 index $=5$

time $=11.0$

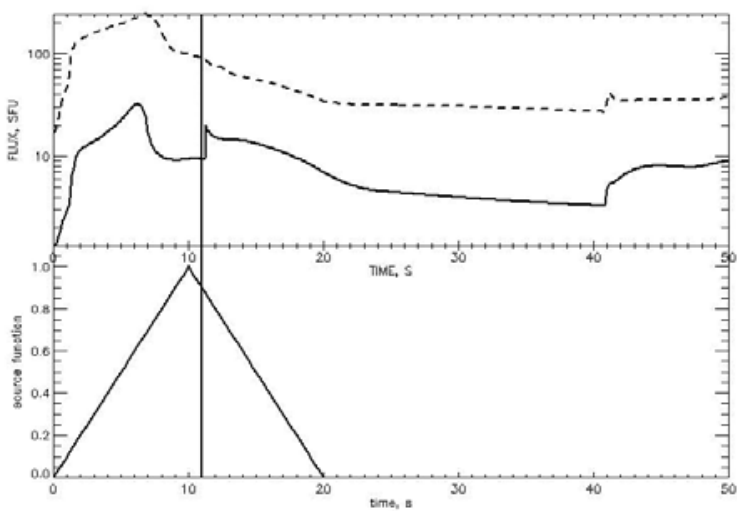

Рис. 1. Левая панель: высотное распределение потока излучения $F(h)$, температуры и концентрации электронов плазмы модельной атмосферы, функции вклада $C F(h)$ источника излучения на частотах 93 (сплошная линия) и 140 ГГц (штриховая линия) в моменты времени $\mathrm{t}=8.5 \mathrm{c}$ (a) и $\mathrm{t}=11 \mathrm{c}($ б). Правая панель: временные профили суб-ТГц излучения на частотах 93 и 140 ГГц и функция инжекции электронов. Вертикальная сплошная линия определяет соответствующие моменты времени, принятые на левой панели. 
На рис. 1 (левая панель) представлены результаты расчетов для двух моментов времени $t=8.5,11 \mathrm{c}$. На высотных профилях можно видеть два ярко выраженных всплеска («антивсплеска») по плотности и температуре, которые соответствуют хромосферным конденсациям. Наиболее интересной особенностью этих образований является то, что они медленно движутся вверх и, как показывают обстоятельные расчеты, вклад конденсаций толщиной всего в несколько десятков километров в суб-ТГц излучение на частотах 93 и 140 ГГц в соответствующие моменты времени является определяющим (см. на рис.1 график высотного распределения потока излучения $F(h)$ и функции вклада $C F)$.

\section{Обсуждение результатов и выводы}

Как следует из расчетов, временной профиль модельного суб-ТГц излучения, в отличие от профиля импульса инжектируемых электронов, имеет два выраженных пика (рис. 1 справа), что связано с неоднородностью плазмы хромосферы и переходной области. При этом максимум суб-ТГц всплеска наступает раньше максимума функции инжекции. Наклон спектра на протяжении всего промежутка времени, длительностью 50 сек, оставался положительным. Образование и релаксация хромосферных конденсаций, характеризующихся пониженной температурой и повышенной плотностью плазмы, сопровождалась существенными изменениями временных профилей суб-ТГц излучения, что, на наш взгляд, может быть использовано для диагностики этих плазменных образований. Это также предполагает заметные различия в поведении временных профилей субТГц и жесткого рентгеновского излучения в пределах десятков секунд, что хорошо согласуется с наблюдениями.

Работа выполнена при поддержке гранта РНФ 20-72-10158 (ГМ) и Минобрнауки (НИР №0831-2019-0006) (ЮЦ, ВС).

\section{Литература}

1. Kostiuk N.D., Pikelner S.B. // Soviet Astron., 1975, 18, 590.

2. Livshits, M.A., Badalian, O.G., Kosovichev, A.G., Katsova, M.M. // Solar Phys., 1981, 73, 269.

3. Kowalski A. F., Allred J.C. // ApJ, 218, 852, 61.

4. Kontar, E.P., Motorina, G.G., and Jeffrey, N.L.S., et al. // Astron. Astrophys., 2018, 620, id A95.

5. Allred J.C., Kowalski A.F., Carlsson M. // Astrophys. J. V. 809. ID. 104. 2015.

6. Vernazza, J.E., Avrett, E., Loeser, R. // Astrophys. J. Suppl. Ser., 1981, vol. 45, pp. 635725 .

7. Morgachev A.S., Yu.T. Tsap, V.V. Smirnova, et al. // Ge\&Ae, in press. 